\title{
AUTOMATIC VISION BASED INSPECTION OF RAILWAY TRACK:
}

\section{A REVIEW}

\author{
Ashwini Belkhade ${ }^{1}$, Snehal Kathale ${ }^{2}$ \\ ${ }^{1}$ Student, ${ }^{2}$ Lecturer, Department of Computer Science and Engineering, GHRIETW, RTMNU, Nagpur, India
}

\begin{abstract}
Currently, most of railway track inspections are manually conducted by railroad track inspectors. Practically, it is not possible to inspect the thousand of miles of railway track by trained human inspector. This inspection takes too much time to inspect the defected railway track and then inform to the railway authority people. In this way it may lead to disaster. Hence to avoid delay and improve the accuracy, our propose system will automatically inspect the railway track by using vision based method and vibration based method. This method proposes continuous monitoring and assessment of the condition of the rail tracks which prevent major disasters. Our proposed system will inspect the rail track component such as missing bolts, tie plates, anchors etc by using vision based method and simultaneously do the calibration of railway track by using vibration based method. The system provides real-time monitoring and structural condition for railway track using vision based method and calibration to search the fault location on the track. Inspections include detecting defects on tracks, missing bolts, anchor, tie plate and clips etc. In vision based method camera we will use to capture the images or videos. In vibration based method some sensors we will use to detect the vibrations on the railway track. We will extract the signal from 2-D.
\end{abstract}

Keywords: Railway track inspection, Vision based and vibration based method, Image processing, Data acquisition.

\section{INTRODUCTION}

Rail track inspection is a necessary task in railway maintenance and is required to periodically inspect the rail track by trained human operator, who is walking along the track \& searching for defects. Such type of monitoring system is unacceptable for slowness and lack of objectivity. This inspection will take too much time to recover from faults. Hence to reduce delay our propose system deals with automatic Visual Inspection of Railway track and devoted to numbers of tasks. Automatic vision based inspection systems is enable to analyze the stipulation of rail track. In this way system increases the efficiency of inspection, reduces the required time and giving a more accurate and frequent information of the railway track. To provide the real time is monitoring and structural condition for railway track using "vision based" and "vibration based" method for safety purpose. In this way we can boost accuracy, efficiency and reliability.

In vision based method our device will capture videos of railway track component using vehicle-mounted Cameras, image enhancement using image processing and assisted automation using a real time tracking algorithms [1].

In vibration based method our device will do calibration of the rail track by using vibration sensors. Vibration sensors will sense the vibration on the track. If $t$ he track vibration are in the range of predefined standard values it means there is no faults otherwise track is defected. Damage component and faulty track information will broadcast to the server through wireless medium i.e. (wifi). By using both the method we can inspect the railway track in accurately. Our propose system focus on machine vision based and vibration based method to detect irregularities in track and defected component such as tie, tie plate, anchor, missing bolts. Inspected data will be stored in digital format that's why analyses of track component condition are possible through data processing. In this way our propose system will perform a function of automatic inspection of track component and calibration of track and it provides an interim improvements to current track inspection practices.

\section{LITERATURE REVIEW}

We first organized a survey of existing technologies of automatic visual inspection of railroad track and track components. This gave understanding with respect to which assignments were best suited to vision based assessment for which technology was not already under development. This survey encompassed well-established inspection technologies and experimental technologies currently under development. In this section, we provide a brief review of the technologies currently in use or development that are of greatest relevance in the determination of the scope of our research.

Safety in railways is one of the major issues for public transportation organization and a fast and efficient inspection system is vital to ensure the safety of railways. Authors had tried to provide effective solution on the problem. Let us 
discuss problem and solution. Previous, rail inspection methods include destructive techniques, such as coring, and non-destructive techniques, such as hammer sounding. But these methods just "cover limited space and have limited effectiveness in identifying the faults. Further non-destructive evaluation techniques for rail track inspection had developed. These technologies include visual inspection, ground penetrating radar (GPR), infrared, X-ray and laser light.

\subsection{Vision Based Inspection Technology}

Automatic railroad track inspection with the help of vision based method. Vision based system there are some cameras for collecting the images or videos of rail track and process the frame image by using image processing. In such manner it could enhance the efficiency of the traditional methods. The System challenges the following addressed: detection, fragmentation, and deformity evaluation of track components whose physically appearance vary across number of tracks and the identification and inspection of track areas such as track turnouts. A MUSIC (multiple signal classification) algorithm is used to detect number of signal in the presence of noise [1].

\subsubsection{Data Acquisition}

In automated visual based inspection of rail track off the shelf cameras are used for obtain rail track images and record this data to a laptop [1]. Digital cameras are used to capture the images or videos of rail track [3]. Surf View comes with on board computer, data acquisition and software along with six cameras scanners and cables [5]A calibrated CCTV camera is used to capture the image frame at resolution $640 \times 480$ at 30 frames per second which was mounted the rail track. The camera will activate and start to obtain video of track [9]. High speed line scan cameras are used to obtain videos of rail track [10].Different types of cameras are used for data acquisition purpose in different vision based system.

\subsubsection{Image Analysis}

The frames of image are proceeds by using algorithm to identify the defected component and assess the stipulation of railway track [3]. The MUSIC algorithm is signal processing technique that extract signal from a 1-D.It provide robust solution against noise and give the accurate result in efficient way. Gabor transformation technique are used to convert into digital signal from track image [1].In vision based system image processing is used to recognize of clips, smoothing and edge detection [8].Machine learning technique is used to process the acquired data [9]. The captured data send to PC with DSP and FPGA boards for real-time frame analysis. Cutting the stream of lines into the frame and then examine frame [10]. Gabor wavelet features is used for frame analysis and it provide a comprehensive result evaluation [11]. To extract rail track image, we first resume evaluating the captured input image with the help of Sobel operator. Hough transform process is used for the detection of railway track lines and removes the noise in the binary image. It is best for detecting straight lines [12]. In feature extraction and recognisation technique, each image frame (from the continuous real time input) is represented by a vector which is made up of color. A different combination of original RGB and $\mathrm{YCrCb}$ is selected for each of the identified sub regions of interest with regarding to both established practice within this area, prior successful classification work within the road domain and related work on other domains where color variance isolation is vital [6].

\subsubsection{Data Analysis}

In this section resulting data compare with the pre loaded standard values, if resulting data lays between the pre specified values for verification purpose [3]. Proposed method is enable to inform the train in case of any dislocations in the track or change in strength of the soil.[2]This system consist of two modules, the first one is the sensor network that monitors the rail track before the train crosses over the railway track and the other is the wireless network that receives the data from the sensor network and inform the corresponding train about the disaster. The vibrations on the track are sensed by these sensors and this method can process the data from the sensor network and inform the train giving sufficient time to stop.The piezoelectric transducer used to sense the vibration of the rail track and activates the sensor network. The sensors placed on both side of the tracks collects the data of the track, the collected data of which are fed to the Operational amplifier based circuit. The output from the piezo-electric transducer (7bits) is feed to an ADC. The digital result from the ADC is given to the encoder circuit which performs the linear block coding and transmits to the destination. The RF receiver is used to receive data. Receiver used in this system AC4490, the range of which is $1.6 \mathrm{Km} \mathrm{[2].}$

\subsection{Detecting Rail Track Component}

In automated visual based inspection system a music algorithm is used [1]. Algorithm is used to detect the rail components. In algorithm a coarse-to-fine approach is used for detecting objects [3].

\subsubsection{Tie Plate and Tie Detection}

Tie plate is placed between the wooden tie and rail to hold the track with the tie. The ties are detected with the help custom filter based on Gabor texture [1]. In tie detection, both shift and spread are used to find out the distance between an anchor and its associated tie [7].

\subsubsection{Anchor Detection}

Anchors are inspected and distance between the tie and tie plate are measured .They are inspected by their parallel edges. Color intensity info is also included for assure that parallel edges have same intensity distributions [3].Use Hough 
transform to detect two horizontal lines in the image, which is horizontal edge of the tie plate. Find the two vertical edges of the tie plate by using Sobel operator. Find the two minimums in the above plot, with respect to the tie plates left and right vertical edge [5]. Multiple cascade classifiers is used to inspect the anchor. For detection purpose a switching modal is used [7].

\subsubsection{Spike Detection}

Two dimensional filters are used for detection purpose [3].The proposed system solved the problem of finding missing clips and finding blue clips which have been placed of damaged rail track [8]. Defects recognition system is continuously monitor the defects before stationary background. The system consists of three modules: a real time track tracking and extraction, emphasize extraction, concealed Markov model (Hmm) preparing, and defects recognition. First, they apply a real-time rail track tracking and extraction algorithm to trace the track and extract the track region, and then they use the Fourier descriptor (FD) to characterize spatial features and the motion analysis to characterize the temporal features [4].

\section{PROPOSED WORK}


Fig 1: Proposed architecture

Our proposed system consists of two models:

1. Vision based automatic tracking of rail track.

2. Vibration based automatic tracking of rail track.

\subsection{Vision Based Method:}

In machine vision, digital camera will use to capture the video. Captured videos or images will process by using machine vision algorithm in image acquisition system and the algorithm will classify the fault according to class. In data analysis the current images compare with the preloaded healthy status of track and verify whether the conditions match or not. If two conditions are matched then there will be no error otherwise the track has some faults. After that this information will broadcast to the server.

\subsection{Vibration Based Method}

In this method a sensors will sense the vibration with the help of vibration sensor and after that it will pass to the op-amp and filter for removing external noise and then it pass to the microcontroller for programming. There is one communication protocol to establish the connection between the hardware and system .In system processing will be done and than it will broadcast to the server through wireless medium. All the procedure is as shown in lower portion of the fig.3.1

\section{CONCLUSIONS}

Our proposed system will automatically inspect the rail track component and calibrations of rail track by using vision based and vibration based method. Our proposed system consist of two parts: A vision based and Vibration based method. In vision based method machine vision algorithm will extract a signal from 2-D signal. In vibration based method will perform the calliberation of rail track. We will perform two tasks at a time so that the system will enhance the capacity of inspection and provides the accurate result. Inspections include tie, tie plates, spikes, anchor etc.

\section{REFERENCES}

[1]. Esther Resendiz, Member, IEEE, John M. Hart, and Narendra Ahuja, Fellow, IEEE "Automated Visual Inspection of Railroad Tracks" IEEE transaction on intelligent transportation systems, vol.14, no.2, June 2013

[2]. Abhisekh Jain, Arvind, Balaji, Ram Viyas N.P.” Onboard dynamic rail track safety monitoring system" International conference on advanced communication systems, January 10 12,2007

[3]. J. Riley Edwards, John M. Hart, Steven Sawadisavi "Advancements in railroad track inspection using machinevision technology" Edwards et al. 2009.

[4]. Feng- Sheng Chen, Chih-Ming Fu, Chung-Lin Huang, " Hand gesture recognition using a real-time tracking method and hidden Markov models" Institute of electrical engineering, national tsing hua university, hsin chu 300,taiwan, roc received 15 January 2001; received in revised form 2 January 2003; accepted 20 March 2003.

[5]. Beena vision "Automated Rail Surface and Track Inspection"

[6]. Isabelle Tang and Toby P. Breckon, "Automatic road environment classification" IEEE transaction on intelligent transportation systems, vol.12, no.2, June 2011.

[7]. Hoang Trinh Norman Haas Ying Li Charles Otto Sharath Pankanti "Enhanced rail component detection and consolidation for rail track inspection" Ibm T. J. watson research center 19 skylikne dr, hawthorne, ny 10532.

[8]. Maneesha Singh, Sameer Singh, "Autonomous rail track inspection using vision based system" IEEE international conference on computational intelligence for homeland security and personal safety alexandria, va, usa, 16-17 October 2006. 
[9]. Avinash Kumar and Narendra Ahuja "A vision system for monitoring intermodal freight train" IEEE workshop appl. computer 2007.

[10]. J. Velton "Image processing algo for real time tracking data".2006

[11]. B.S. Manjunathi and W.Y. Mab, "Texture Features for Browsing and Retrieval of Image Data" IEEE transaction on pattern analysis and machine intelligence, vol.18, no.8, August 1996.

[12]. Fatih Kaleli and Yusuf Sinan Akgul , "Vision-Based Railroad Track Extraction Using Dynamic Programming" proceedings of the 12th international IEEE conference on intelligent transportation systems, st. louis, mo, USA, October 3-7, 2009.

\section{BIOGRAPHIES:}

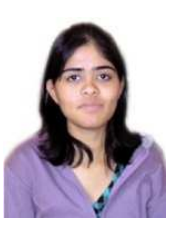

First Author: Ashwini Belkhade has received B.E. in the field of Electronics \& Telecommunication from Rashtra Santa Tukadoji Maharaj Nagpur University (RTMNU) in the year 2011. She is pursuing M.E. in Wireless Communication and Computing from G.H.Raisoni Institute of Engineering and Technology for Women Nagpur. Her research interests includes Wireless Communication and Image Processing.

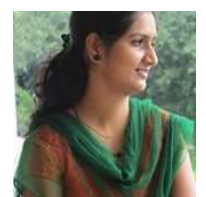

Second Author: Snehal Kathale is a lecturer in Dept. of Computer Science at G. H. Raisoni Institute of Engineering and Technology for Women, Nagpur. 